\title{
Miscellanées : Itinéraire d'un « grand chef » kanak indépendantiste
}

À propos de Nidoïsh Naisseline. De cœur à cœur. Entretiens

Isabelle Leblic

\section{CpenEdition}

\section{Journals}

Édition électronique

URL : http://journals.openedition.org/jso/7855

DOI : $10.4000 /$ jso.7855

ISSN : $1760-7256$

\section{Éditeur}

Société des océanistes

Édition imprimée

Date de publication : 15 décembre 2017

Pagination : 345-352

ISSN : 0300-953x

Référence électronique

Isabelle Leblic, « Miscellanées : Itinéraire d'un « grand chef » kanak indépendantiste », Journal de la Société des Océanistes [En ligne], 144-145 | 2017, mis en ligne le 15 décembre 2017, consulté le 15 mars 2021. URL : http://journals.openedition.org/jso/7855 ; DOI : https://doi.org/10.4000/jso.7855

\section{(c) $\oplus \Theta \Theta$}

Journal de la société des océanistes est mis à disposition selon les termes de la Licence Creative Commons Attribution - Pas d'Utilisation Commerciale - Pas de Modification 4.0 International. 


\section{MISCELLANÉES}

\section{Itinéraire d'un " grand chef » kanak indépendantiste. À propos de Nidoïsh Naisseline. De cour à cour. Entretiens}

par

Isabelle LEBLIC ${ }^{*}$

À propos de: Kotra Walles, 2016. Nidoïh Naisseline. De cœur à cour. Entretiens, Papeete, Au vent des îles, 204 p., 17 photos noir et blanc.

Ce troisième ouvrage d'entretiens menés par Walles Kotra avec des personnalités calédoniennes, après Jacques Lafleur et Antoine Kombouaré, nous emmène cette fois-ci à Maré avec le militant kanak et grand chef Nidoïsh Naisseline (1945-2015). En se faisant ainsi passeur de parole, le journaliste kanak originaire de Tiga qu'est Walles Kotra nous présente tour à tour divers aspects de la société calédonienne.

Dans la préface (pp. 9-14), Walles Kotra raconte et analyse sa rencontre avec le grand chef Naisseline :

«Il vous démontre, textes à l'appui, l'apport de Ronsard et Du Bellay dans son combat pour la décolonisation culturelle kanak. Et lorsque vous l'interpellez sur son engagement pour l'indépendance, il fait un détour par De Gaulle et son fameux discours de Brazzaville. Une question sur l'identité kanak et il

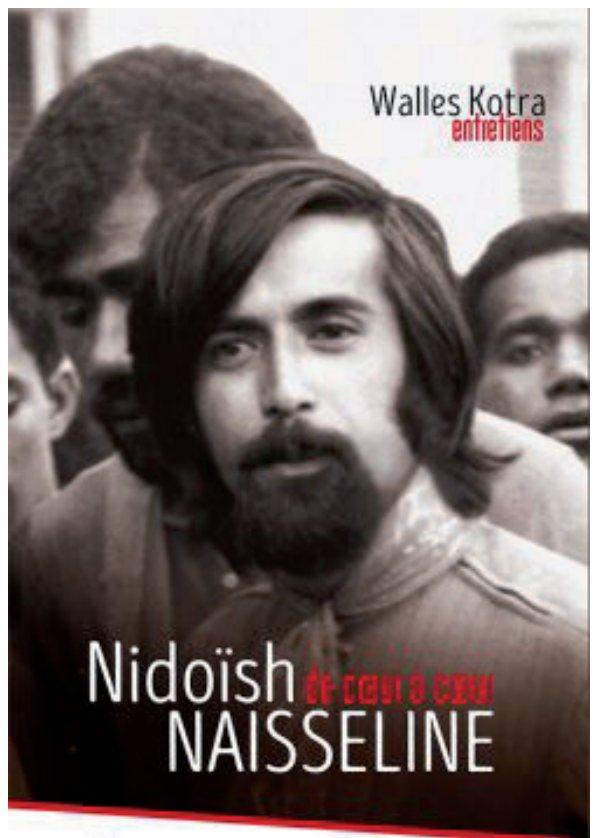

Nuveroesites

1. Il doit à une enseignante, Mme Ougaud, d'avoir découvert «le mouvement de la Renaissance en France. [...] la volonté des Français d'arrêter de copier l'extérieur et de revenir chez eux. De trouver en eux inspiration et force. [...] Ce poème [Heureux qui comme Ulysse de Du Bellay] m’a toujours accompagné et, en particulier, pendant la période des Foulards rouges où, pour la première fois, nous utilisions l'expression "beauté kanak". " (p. 18). À ce propos, il précise plus loin que «être fier de ce que l’on est, c'est assez révolutionnaire pour un colonisé » (p. 37).

* Anthropologue, cNRs LAcito à Villejuif, leblic@vjf.cnrs.fr 
convoque Levinas et Jankélévitch. Une interrogation sur l'avenir et il raconte l'histoire du vieux Cuk de Menaku ou rappelle une citation du vieux Abraham Manace $^{2}$ de Lece. » (pp. 11-12)

C'est donc tout un programme que l'itinéraire d'homme et de chef kanak de Nidoïsh Naisseline, qui a traversé son époque, et que ce livre nous donne à découvrir « en suivant simplement sa parole» (p. 12) et on apprend beaucoup de choses sur ce parcours méconnu de beaucoup. D'ailleurs, on ne peut s'empêcher de lire ce livre sans penser au film de Nunë Luepack, Nidoïsh Naisseline: une parole qui ne meurt pas (http:// www.sanosi-productions.com/projet/nidoishnaisseline), que j'ai vu à Rochefort Pacific en mars 2016 en même temps que le très beau film de Deborah Walker et Neil Morrisson sur Déwé Gorodé : Écrire le pays. Comme un pied de nez de l'histoire, ces deux films ont été projetés la même année alors que tous deux avaient milité ensemble aux Foulards rouges, Déwé étant la cadette de Nidoïsh de trois ans, et avaient été emprisonnés en même temps (voir la photo p. 123 les montrant tous deux au pied du tribunal lors de leur procès du 5 novembre 1976). Autre parallèle, l'un est du côté de la parole, de l'oralité alors que Déwé a choisi de s'exprimer par ses écrits, que ce soit des poèmes ou des romans, peut-être parce qu'il était aussi plus difficile à une femme de prendre la parole à cette époque ? Nous y reviendrons ci-dessous.

Walles Kotra, auteur de ce livre, précise :

"Je ne suis ni son biographe ni son porte-parole coutumier. J'ai simplement voulu, avec son accord, mettre par écrit son témoignage sur son pays et son époque. [...] Nidoïsh Naisseline [...] explore une question qui l'obsède mais qui est vieille comme l'humanité : comment construire un pays ensemble? Autour de quelles valeurs? Avec quels mots et quels regards?»(p. 14)

Suivent douze chapitres que l'on peut dire thématiques plus que chronologiques car ils reprennent tant des moments de la vie du Grand chef - par exemple, son éducation de chef ou sa scolarité à Paris - que des événements qui l'ont rythmée - comme le naufrage de La Monique, la naissance des Foulards rouges... - et une postface de Walles dans l'avion de retour de Maré.

Parmi les drames qui ont touché l'île de Maré et sa population, on peut noter le naufrage de La Monique, l'épidémie de lèpre, les morts dans les deux conflits mondiaux et, plus récemment, les affrontements de Maré de 2012. Toute mort violente en société kanak marque les esprits, surtout quand celles-ci se produisent en nombre. Comme aimait à le dire Jean-Marie Tjibaou, «à l'échelle de notre petit peuple, les morts qui se produisent collectivement représentent un véritable traumatisme", comme ce fut le cas dans l'embuscade de Tiendanite le 5 décembre 1984, l'exécution d'Éloi Machoro et de Marcel Nonnaro le 12 janvier 1985 (comm. pers., à Paris, en février 1988), ou encore plus tard, avec les dixneuf morts de la grotte d'Ouvéa le 5 mai $1988 . .$.

À l'occasion du soixantième anniversaire de la disparition des cent vingt-six victimes du naufrage du caboteur La Monique en 1953 - Nidoïsh avait alors 8 ans -, devant le monument de Tadine, Nidoïsh Naisseline revient sur la blessure collective que cet événement a représenté et c'est ainsi que commence le récit (chapitre 1, pp. 15-20). Ce naufrage se produit au sortir de l'indigénat, au moment où :

«il fallait presque se repositionner, se relever. Le système colonial avait réussi non seulement à nous enfermer dans les réserves mais également dans nos têtes. [...] Il fallait sortir de tout cela et redevenir sereinement soi-même. Ce mouvement a été amplifié ensuite par Mélanésia 2000 et Jean-Marie Tjibaou. [...]J'ai été [...] étonné et fier de découvrir que ce que me disaient certains de mes vieux à Maré, je pouvais le retrouver dans des écrits de Confucius. J'ai pris conscience de la part universelle de notre culture. » (p. 19)

Puis, dans le chapitre 6 intitulé "Bessadra" (pp. 103-108), du nom de la léproserie de Maré, il revient sur l'épidémie de lèpre qui a touché lourdement l'île comme l'ensemble des Loyauté et sur les engagés kanak de Maré tués pendant la Première et Deuxième Guerre mondiales. Ce qui a été le plus dur, nous dit Nidoïsh, ce n'est pas tant la maladie en elle-même que l'isolement dans lequel les malades avaient été placés car « les gens étaient là sans être là » (p. 103). Et, dans une société où tout repose sur les relations, cette mise à l'écart représentait donc un grand traumatisme, pour les malades comme pour leurs familles. D'ailleurs, il le mentionne lui-même quand il parle de la "vingtaine [de morts de la Seconde Guerre mondiale], et à l'échelle du district, c'était lourd» (p. 108).

Enfin, dans le chapitre 7 (pp. 109-116), Nidoïsh Naisseline parle des événements de Maré de 2011-2012 qui ont fait quatre morts suite au blocage de l'aérodrome de La Roche alors qu'il était président du conseil d'administration d'Air Calédonie. Un conflit eut lieu pour lutter contre la hausse des tarifs. Un comité des usagers créé pour l'occasion, suivi par l'USTKE et le Parti travailliste, le rendait responsable de ces augmentations tarifaires.

« À Maré, les choses ont pris une autre tournure parce que cette crise a cristallisé d'autres conflits qui 
étaient sous-jacents : coutumiers, religieux et même politiques. [...] Sur le plan coutumier, le mouvement s'est vite situé en opposition et en rivalité entre la chefferie de Guahma et une coalition intitulée "Elenod" qui regroupait les quatre chefferies catholiques de La Roche. » (pp. 110-111)

En réaction, se constitue un autre comité, qui ne voulait pas que les "grèves d'Air Calédonie avec une prise en otage de la population des îles " recommencent, qui rassembla également des défenseurs de la chefferie de Guahma attaquée.

Une part importante du récit présente l'éducation du jeune Nidoïsh et du futur chef. Dans le chapitre 2, "L'éducation d'un chef. "les paroles et les silences" " (pp. 21-43), il nous explique :

" qu'une chefferie, c'est d'abord la responsabilité de servir. Ce n'est pas une structure féodale [...] Ce n'est pas un pouvoir, c'est un service. C'est une sorte d'obligation et une personne n'est pleinement humaine que lorsqu'elle remplit ses obligations. [...] Dans les petites sociétés insulaires d'Océanie, la légitimité ne peut pas se fonder uniquement sur la force. Elle doit reposer sur un consensus et c'est un équilibre fragile. » (p. 22)

Ce qui lui fait dire que :

"Être chef, c'est la capacité de représenter le groupe » (p. 24)

Et en parlant de son éducation kanak, il précise que :

" [un] point important, c'est tout ce qui touche à la gestion de la relation à l'autre » (p. 24)

et qu'en tant que futur chef,

"il fallait prendre le temps, apprendre à écouter, choisir les mots et le moment pour bien gérer les hommes. » (p. 25)

Au passage, il rappelle, et je ne peux qu'être d'accord avec cela, que les ethnologues ne sont ni là pour donner des leçons ni pour prétendre en savoir plus que les Kanak eux-mêmes (ce qui est une tendance chez certains collègues il est vrai) :

« Ils [les sages kanak] n'ont pas besoin que les ethnologues leur expliquent leur culture. » (p. 27)

"Il faut faire attention à l'influence des ethnologues même s'il y a des exceptions comme Guiart ou d'autres. J'ai eu beaucoup de débats avec eux parce que, quand ils parlent des choses de chez nous, quand ils écrivent des idioties, cela n’a aucune conséquence pour eux et leurs instituts de recherche à Paris. [...] Mais ici cela peut souvent provoquer des drames dans les familles et les clans. Il y a récemment une enquête qui a été menée en milieu kanak et ils ont fini par écrire que nous cautionnons le viol des femmes. C'est tout à fait scandaleux. Dans nos petites sociétés insulaires, il faut faire attention à l'endroit où l'on met les pieds. » (p. 179)

Il revient sur l'engagement gaulliste de son père et sur le fait qu'il "a fini par voter indépendantiste » tout en restant gaulliste (p. 33). Il rappelle à juste titre que le gaullisme, pour les Kanak de cette époque, c'était la résistance durant la $\mathrm{Se}$ conde Guerre mondiale et le discours de Brazzaville du général De Gaulle en 1944 où :

«il avait proclamé la nécessaire émancipation des peuples d'outre-mer. » (p. 33)

Après l'école élémentaire à Maré, il part au collège Surleau à Nouméa en 1953 avec son père, sur son cotre Dada, en faisant " escale à Goro dans le Sud [...] [car] c'est un peu le chemin que devaient emprunter les gens des îles et en particulier de Maré qui arrivaient sur la GrandeTerre» (p. 41)

Au collège Surleau, il a vécu la mise à l'écart des collégiens vietnamiens suite à Diên Biên Phu. Puis, après le lycée La Pérouse où il fit la rencontre d'Élie Poigoune ${ }^{3}$ qui y enseignait, "grand professeur de mathématiques » et " actuellement présidente de la Ligue calédonienne des droits de l'Homme ", il partit en France poursuivre ses études au lycée Jean-Jacques Rousseau de Montmorency en 1962 à la fin de la guerre d'Algérie. Le proviseur de ce lycée était le gendre de Maurice Leenhardt, Éric Dardel, dont il découvrit plus tard qu'il était "l'un des pères [...] de la "géographie culturelle" » et que c'est cet homme qui l'a " vraiment amené au livre »:

«[...] il m'a appris que le livre est essentiel pour se construire dans la vie et pas seulement pour ses études. " (p. 37)

\section{Alors que :}

"La coutume, c'est tout à fait autre chose. C'est ce qui donne un sens à la vie et à la vie en société. » (p. 38)

Le chapitre 3 «Paris» (pp. 45-66) où il a passé son bac est résumé en trois mots "Étudier, s'engager et rencontrer ». Sa sœur aînée, Sophie, qui était une élève brillante, n’avait pas été réadmisse à La Pérouse suite à une hospitalisation, alors qu'elle était guérie.

"Dans la famille, nous avons ressenti cela comme une injustice. Alors, lorsqu'en 1962, j'étais admis en seconde, plusieurs personnalités locales comme Jean Guiart, Maurice Lenormand ou encore le vieux Roch Pidjot ont conseillé à mon père de me faire partir en

3. Il ne nous dit pas qu'il était aussi l'un des fondateurs du Palika (voir à ce propos l'article de B. Trépied dans ce numéro, pp. 239-252). 


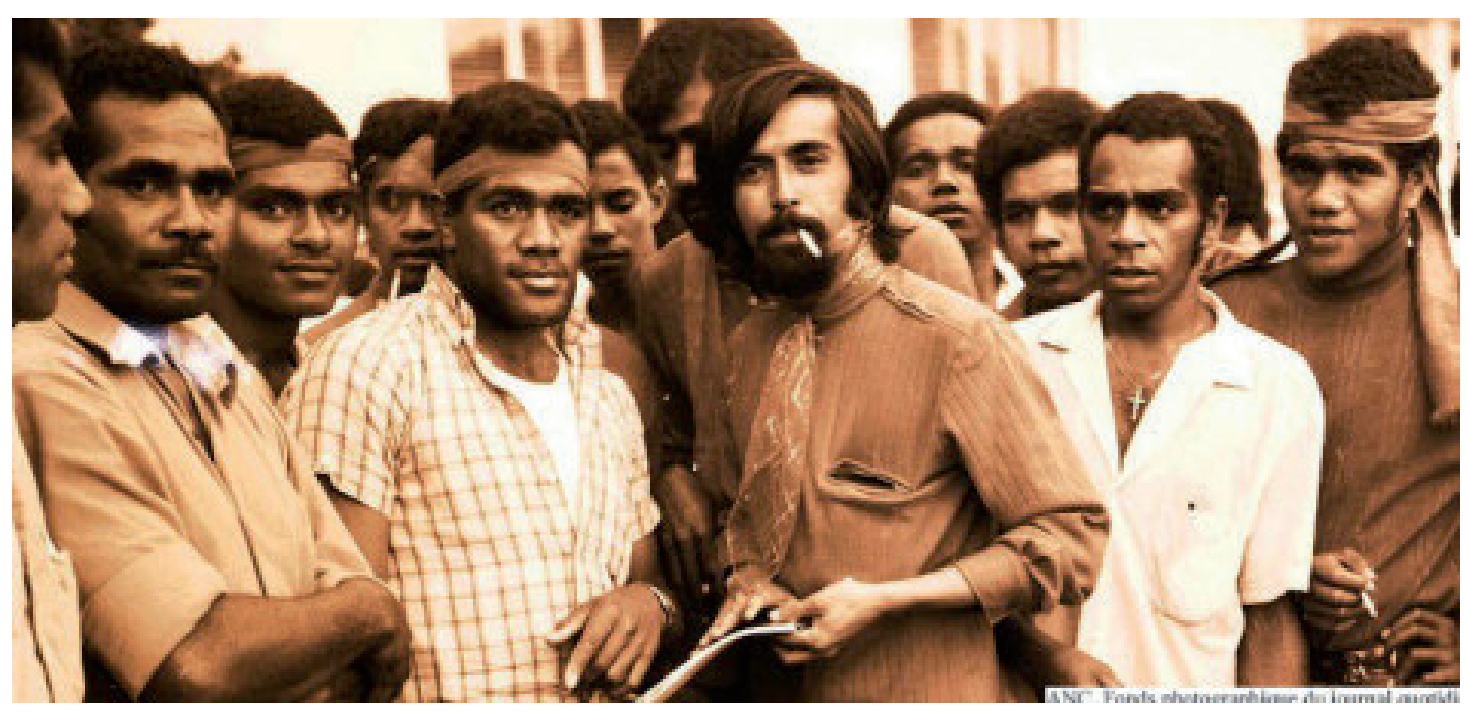

Рното 1. - Nidoïsh Naisseline à l'époque du procès des Foulards rouges (C) Fonds photo de La France Australe - 113Fi-5387, archives de la Nouvelle-Calédonie, reproduite p. 120 du livre)

France pour éviter que ce genre d'histoire ne se reproduise. » (p. 38)

Ce départ en France à 17 ans, organisé par l’Église évangélique dont il nous dit que :

«Sur le plan politique, ils étaient souvent engagés à gauche et très clairement colonialistes » (p. 47),

a sans doute pesé de tout son poids dans la formation intellectuelle de Nidoïsh, notamment par le fait de le sortir de son cocon de futur chef kanak :

«Heureusement, je suis parti en France pour continuer mes études. Le cocon a fini par exploser et j'ai pu un peu plus me défouler et respirer.» (p. 30)

Le proviseur Éric Dardel, gendre de Maurice Leenhardt, marqua profondément le jeune Naisseline :

«Dardel conceptualisait ce que nous vivions tous les jours à la tribu. Sa vision cassait un peu le rationalisme occidental et réintroduisait le sacré dans notre relation à notre environnement. [...] Les conversation avec Dardel m’ont passionné et aidé. » (p. 46)

\section{En France, il fut accueilli par Mme Weben ${ }^{4}$ :}

«femme engagée qui avait pris fait et cause pour le monde kanak. Après le drame d'Ouvéa et l'attaque de la grotte de Gossanah en 1988, elle a voulu être la marraine de Benoît Tangopi, emprisonné à Paris. Elle lui écrivait et lui envoyait des colis en prison. Elle l'a suivi jusqu'à sa libération après l'amnistie décidée par les accords de Matignon. » (p. 49)

\section{La sœur de Mme Weben était l'épouse de Charles} Veillon :

"grand commerçant suisse basé à Lausanne [...] grand mécène, qui, par le biais de sa Fondation, accompagne des écrivains et des artistes. [...] Édouard Glissant a d'ailleurs reçu le prix Veillon du roman en 1965 pour son ouvrage Le quatrième siècle $e^{5}$. (p. 47)

Cette femme qu'il appelait " mamie " a beaucoup compté dans sa vie, que ce soit durant ses études ou après, avec la création des Foulards rouges et le procès qui s'ensuivit après son incarcération en 1969. Ce fut le début d'une nouvelle rencontre importante, tant du point de vue personnel que pour le peuple kanak en général. C'est maître Jean-Jacques de Félice ${ }^{6}$, lui aussi élevé dans une culture et un milieu protestant, qui fut contacté à la demande de Nidoïsh et de son père par Mme Weben pour venir assurer sa défense à Nouméa. Il précise d'ailleurs que c'est $\mathrm{M}^{\mathrm{e}}$ De Félice :

4. Mme Weben, comme maître De Félice (voir ci-dessous), s'est investie dans le soutien aux Kanak et à l'indépendance qui s'est développé en France métropolitaine dans les années 1980.

5. Rappelons que ce roman est une "véritable somme de l'itinéraire historique de la société coloniale [qui] inverse la geste de la colonisation par la quête des traces originelles de la société antillaise, pistée en ses non-dits. [...] Le récit a constitué un tournant décisif, non seulement dans l'œuvre de Glissant, mais aussi dans l'inspiration même de la littérature dite postcoloniale. » (http://www.fabula.org/actualites/le-quatrieme-siecle-d-edouard-glissant-50-ans-apres-site-officieledouard-glissantfr_66400.php).

6. Né le 15 mai 1928 et décédé le 27 juillet 2008, Me Jean-Jacques de Félice fut un grand défenseurs des opprimés de toute sorte (Algériens du FLN, paysans du Larzac, SDF parisiens...). Il fut parmi les créateurs de l'Association information et soutien aux droits du peuple kanak (AISDPK) en 1981 et en assuma la présidence pendant de nombreuses années. 
" qui a été pour [lui], et pour nous, le visage de cette France que l'on aime » (p. 66),

phrase que tous les militants anticolonialistes que nous sommes, quand nous nous rendons en terrain kanak, avons entendue régulièrement dans la bouche de nos hôtes kanak. D'ailleurs, à propos de ce milieu protestant dans lequel il a évolué très jeune en France, Nidoïsh Naisseline précise que :

"sur le fond, en tant que protestant, je me suis d'emblée situé dans le camp de ceux qui luttent pour la décolonisation. » (p. 54)

\section{Et il ajoute ensuite qu'il a été aussi :}

"profondément marqué [...] par la découverte et la lecture de Frantz Fanon et notamment de ses deux livres incontournables: Peau noire, masques blancs et Les Damnés de la terre." (p. 55)

Il rappelle d'ailleurs qu'il a publié en NouvelleCalédonie :

"sous son nom, un plagiat [...] d'un paragraphe [du premier livre que] Max Chivot a intitulé "Aspects noir d'un problème blanc". J'ai juste mis Kanak à la place de nègre et quelques adaptations ici ou là [...]. C'était la première fois qu'un Kanak dénonçait publiquement la colonisation aussi clairement et cela a marqué les gens. En réalité, j’avais triché ; c'étaient les mots de Fanon. » (p. 55)

Puis, dans les chapitres suivants, Nidoish Naisseline revient sur sa vie au pays, à Nouméa dans le chapitre 4 (pp. 67-76) depuis 1953, sa première venue dans la capitale calédonienne, et dans le district de Guahma à Maré dans le chapitre suivant (pp. 77-102). À propos de Nouméa, il précise :

« Finalement, contrairement à ce que l'on craignait, Nouméa n’a pas fait disparaitre nos coutumes. Les hommes arrivent toujours à s'exprimer et à positiver. » (p. 72)

Le chapitre 5 est sous-titré " Naisseline ne Warawi » (p. 77), "double représentation qui identifie le district " (ce qui signifie en substance l'ensemble de la population du district de Guahma). Dans ces quelques trente pages, il nous explique le fonctionnement de la société maréenne à travers l'exemple de sa chefferie, chapitre des plus instructifs pour tout ethnologue. Ici encore, il marque son désaccord avec la pratique de certains chercheurs ou journalistes qui :
" au nom de la recherche scientifique ou de la liberté de la presse $[\ldots]$ se permette[nt] de ne pas respecter les règles $[\ldots] »($ p. 90$)$.

Dont acte ! Mais je partage cet avis à plus d'un titre... car certains de nos collègues se permettent encore aujourd'hui de donner des leçons au Kanak, comme au bon vieux temps de l'ethnologie coloniale ou font preuve de points de vue très étnocentrés!

Quand Walles Kotra l'interroge sur l'avenir des coutumes kanak, il répond :

"Ce que j'observe, c'est qu'il y a plutôt un réveil de la coutume. Je vois comment Cas [son fils qui prend sa succession en tant que chef] fonctionne. Il y a chez les gens une volonté d'affirmation culturelle forte. » (p. 96)

Ici encore, il s'en prend indirectement aux chercheurs quand à la position et aux rôles de femmes kanak :

"C'est important, au moment où l'on débat beaucoup du statut de la femme, que nous mesurions la place et le rôle de ces femmes-là. On ne trouve pas cela dans les livres ou dans les colloques mais c'est quelque chose de fondamental dans notre culture. Parce que ces femmes sont la parole. Pas la parole de cérémonie. Pas les discours. La parole de la vie et c'est ce qui est essentiel. » (p. 98, souligné par moi)

Les quatre derniers chapitres reviennent sur l'itinéraire politique du grand chef Nidoïsh Naisseline. Nous l'avons déjà évoqué ci-dessus à l'occasion de ses rencontres avec des personnalités qui l'ont grandement influencé (Mme Weben, maître De Félice...). Mais c'est aussi Mai 68 qui eut sur lui, comme sur beaucoup de jeunes Kanak étudiant en France, une importance déterminante. Dans le chapitre 8 (pp. 117-129), il nous raconte le contexte de la création des "Foulards rouges" qu'il qualifie de " parfum de la beauté ", en plein boom du nickel, dans cette période de transformation de la société calédonienne qui voyait les Kanak voter depuis peu, qui avait même des élus Kanak dont le député Roch Pidjot, période où l'Union calédonienne était encore largement majoritaire (Leblic, 1993 : 59).

«La colonisation est inséparable de la notion de blasphème. [...] Le pire, c'est que nous finissions par reproduire, par mimétisme, cette vision coloniale du monde en négligeant la beauté qui est en nous. [...] Les Foulards rouges, ce fut d'abord une prise de conscience de ce blasphème et ensuite, une volonté de la jeunesse de le faire voler en éclats et de redécouvrir ainsi affectivement, esthétiquement et politiquement sa propose culture. » (pp. 117-118)

7. Cette alliance entre la FNSC et le fi qui permit à Jean-Marie Tjibaou de devenir en 1982 président du gouvernement de Nouvelle-Calédonie fut précusseur et permit, entre autres choses, la tenue de la table ronde de Nainvilles-les-Roches en juillet 1983. 
Les " Foulards rouges", qui n'est pas comme un mouvement politique, mais qu'il caractérise plus comme un regroupement d'individus très différents tels que, entre 1969 et 1971, Déwé Gorodé, Max Chivot, Fote Trolue, Susanne Ounei, Louis Kotra Uregei, Franck Wahuzue, le pasteur Wakaine... et d'autres étudiants calédoniens de Paris, qui discutaient, partageant une vision commune, des poèmes, des chansons, des lectures et des textes qu'ils écrivaient. C'était, dit-il (p. 120), un mouvement de jeunes assez insaisissables qui fut qualifié "Foulards rouges" par l'extérieur (Gaby Briault dans son journal Nymakan). C'était un mouvement littéraire ou artistique (p. 121) - dont la président était Dewe Gorodé -, qui « a marqué la résurrection inattendue de l'univers kanak [...] comme si une petite lumière s'était allumée dans la nuit pour rappeler que nous existions et dire notre vérité » (p. 123).

"À cette époque, le manou et le foulard étaient assez souvent portés dans les tribus. Et on s'est aperçu que eux qui avaient le foulard rouge étaient souvent arrêtés par la police pour des contrôles divers. Les jeunes ont donc décidé de l'assumer, de le revendiquer et c'est ainsi que c'est tout simplement imposée la couleur. » (p. 119)

À cette époque, le racisme de la ville blanche fait rage et c'est dans ce contexte colonial s'il en est qu'intervient l'affaire du tract en langues kanak. Fote Trolue, accompagné de deux ou trois autres jeunes, se fait refuser l'entrée d'un restaurant de la baie des Citrons et décide d'écrire un trac pour protester contre cette attitude raciste.

" Il le traduit en drehu (lifou), Yeiwene traduit ce tract en nengone (maré) et nous le distribuons. [...] Il y avait alors à Nouméa comme une psychose, une peur de l'insurrection ou de la descente des Kanak sur la ville. La machine répressive s'est donc mise en mouvement. »(p. 119)

S'ensuivent manifestions, arrestations, prise d'assaut du commissariat central... et :

" c'est parti ensuite comme une traînée de poudre en cette année 1969. Je me retrouve en prison avec d'autres. Maittre De Felice, l'avocat de Che Guevara, intervient pour nous défendre, une chanson est composée, Yann Celene Uregei et d'autres élus kanak quittent l'Union calédonienne et créent l'Union multiraciale avec des hommes comme Wakolo Pouyé, Caba Caba, Willy Nemia, Edmond Nekiriai ou encore James Wright. Les Foulards rouges, ce mouvement né de nulle part, a eu des répercussions politiques importantes et, fait nouveau, les jeunes se sont mobilisés. Ils n'ont plus peur de prendre la parole et de descendre dans la rue. » (p. 120-121)

"Mon chemin politique. "La richesse des pauvres" " est le titre du neuvième chapitre (pp. 131-153) qui retrace également sa vie d'élu dans diverses instances politiques : à l'assemblée territoriale, à la province des Iles, au congrès du territoire. Il revient (p. 134) sur la courte mais fondatrice expérience du gouvernement Tjibaou (1982-1984) : c'était la première coalition entre des indépendantistes et des non-indépendantistes comme Jean-Pierre Aïfa, Gaston Morlet ou Stanley Camerlynck, membres de la Fédération pour une nouvelle société calédonienne ( $\left.\mathrm{FNSC}^{7}\right)$ avec qui le Front indépendantiste (FI) s'est allié pour prendre le gouvernement, sans oublier Henri Wetta, membre du RPCR qui a rejoint la coalition ; c'était aussi la première fois que les Kanak étaient majoritaires dans un gouvernement ; c'était aussi la première fois qu'une femme y participait en la personne d'Yvonne Hnada du Parti socialiste calédonien (PSC). Ce n'était "pas une simple coalition. C'est un pays qui se mettait en mouvement parce qu'il y avait un profond respect et des échanges très riches " (p. 135). Côté réalisation, ce gouvernement n'est pas en reste non plus : mise en place de l'impôt sur le revenu, création d'Air Calédonie international, Fonds d'aide au développement de l'intérieur et des îles (FADIL), réforme foncière ou encore prise en compte des langues kanak à l'école... :

" Je crois que nous avons fait la preuve que nous pouvions passer d'une culture d'opposition à une culture de gestion et cela, presque naturellement. » (p. 134)

Et cela nous rappelle, s'il en était besoin, que les accords n'étaient pas si révolutionnaires que cela, et comme j'aime à le dire et à l'écrire, une grande part de ce qui était contenu dans les accords de 1988 existait déjà depuis de nombreuses années mais sous un autre nom. Depuis cette coalition de gouvernement de 1982, puis la table ronde de Nainville-les-Roches de juillet 1983, la plupart des choses qui se trouvent dans les accords était déjà présente en filigrane: le rééquilibrage, la Promotion mélanésienne ${ }^{8}$ comme on l'appelait à l'époque, le destin commun avec l'accueil des victimes de l'histoire, etc.

Au passage, Nidoïsh Naisseline revient sur les illusions kanak quant aux socialistes français :

«Nous avions tout simplement confiance dans le Parti socialiste français. Malgré ce qui s'est passé dans l'histoire et notamment en Algérie, nous pensions que les socialistes de 1981 étaient des anticolonialistes qui allaient tout faire pour nous accompagner. [...] Je crois qu'on s'est trompés. [...] Mais le parti était souvent dans le calcul politicien où les postures nationales, la rivalité avec la droite en France, étaient plus importantes que l'avenir des Kanak et des Calédoniens. Ils ont même essayé d'intervenir directement chez nous en instrumentalisant tel ou tel. » (p. 135-136) 
Bien sûr, il revient sur le "traumatisme" des "Événements" qui ont secoué le pays, ceux de 1984-85 et ceux d'Ouvéa, qui sont liés au fait que :

« Avec Yeiwene et d'autres, on partageait ce sentiment d'être humilié chez soi, dans son propre pays. Mais en même temps, nous avons vu aussi pendant cette période que notre peuple pouvait se lever. La jeunesse mais également nos anciens, les mamans, les associations, les Églises, tout le monde kanak pouvait se dresser pour dire qu'il ne se laisserait jamais faire. » (p. 136)

Et c'est exactement ce que j'ai connu dans les années 1985-1986, une mobilisation générale de l'intérieur et des îles pour réclamer l'indépendance kanak. Pour la jeune ethnologue que j'étais, lors de mon deuxième séjour de terrain en milieu kanak, cette période fut d'une richesse jamais inégalée dans les rencontres, discussions de toutes sortes, tant sur mon propre travail que pour le militantisme anticolonialiste (voir Leblic, 1993, 2004).

Il revient aussi sur la "tragédie d'Ouvéa " :

"Nous étions désemparés. Nous avions des relations, y compris coutumières, avec Djubelly Wea. Lorsque tout s'est déclenché, j'ai essayé de l'appeler mais les communications ont été coupées avec Ouvéa. J'étais à Maré et en contact avec Yeiwene Yeiwene. Au bout d'un moment, l'enchaînement des événements devenait incontrôlable et tout a été emporté comme lors du passage d'un cyclone. On était allés trop loin. Nous avons tous été profondément meurtris par $\mathrm{Ou}$ véa et la blessure est encore là. » (p. 137)

Mais il ne dit rien malheureusement des excès de l'armée à Ouvéa qui elle est allée encore plus loin... bien qu'il précise simplement par la suite, que :

" il était difficile de nous appuyer sur un Mitterrand qui, quoi qu'il dise, a donné l'ordre de l'assaut de la grotte. » (p. 193)

Puis il parle des accords de Matignon auxquels il a participé à la demande de Jean-Marie Tjibaou :

" Je crois qu'il pressentait déjà à ce moment-là que le plus dur ne serait pas seulement la négociation et la signature d'un accord mais ce qui allait se passer après, lorsque nous rentrerions avec les accords au pays. Il savait, et la suite malheureusement le confirmera, que ce serait difficile de faire partager à notre population ces accords ${ }^{9}$ parce qu'ils représentaient une rupture par rapport à notre combat et notre stratégie. " (p. 138)

À une question de Walles qui lui demande de revenir sur sa carrière politique, Nidoïsh répond :
" on a l'impression de tourner en rond et de redire les mêmes choses depuis trente ans " (p. 143),

phrase que j'ai souvent entendue en 1984-88 dans la bouche de Jean-Marie Tjibaou quand il revenait de rencontres avec les hommes politiques français.

Les chapitres 10 "Notre identité - Kanak et Calédonie» (pp. 155-162) et 11 "Ce que je crois » (pp. 163-190) reviennent sur son itinéraire de militant et de Grand chef. Il parle aussi de tous ceux qui ont croisé son chemin, en pays kanak ou ailleurs... À propos de l'identité kanak et de l'avenir du pays, il précise que :

«Si nous ne partons pas de cette référence à la terre, ce sera difficile de construire une citoyenneté. C'est pareil pour les Caldoches. Finalement, leurs racines sont également ici et il faut partir de là. Et notre combat, depuis les Foulards rouges, c'est de dire qu'il faut être fier de ce que nous sommes. Fier d'appartenir à cette terre et à ce pays. Et à partir de ce constat, il faut partager de cœur à cœur. On peut le faire d'autant plus que la terre nous réunit et nous protège. » (p. 168)

Mais est-ce que cette vision, déjà mise en avant par les Kanak à Nainville-les-Roches en juillet 1983, est partagée par tous les habitants du Caillou? Rien de moins sûr malheureusement. D'ailleurs, il précise que :

"La difficulté, c'est que, pour l'avenir, il ne suffit pas de trouver une solution politique. [...] L'avenir, c'est surtout la façon de partager les mêmes valeurs. » (p. 195)

\section{Et c'est sans doute là que le bât blesse !}

Dans le chapitre 12 «Et demain? partager notre terre" (pp. 191-198), Nidoïsh réaffirme que " pour fonder notre vivre ensemble ", il faut d'abord reprendre possession de sa propre terre afin de ne plus être "des étrangers chez nous " (p. 191), comme cela avait déjà été affirmé depuis Nainville avec les victimes de l'histoire :

"Effectivement, lors des accords, nous avons dit: "Ici c'est chez nous, venez partager ce pays avec nous". " (p. 192)

"Il y a un préalable qu'il faut bien respecter, c'est la notion de peuple indigène. [...] C'est pour cela qu'il est impératif de maîtriser l'immigration et de ne pas nous noyer dans une population venue d'ailleurs. [...] Nous devons nous référer aux recommandations des Nations unies sur cette question-là. » (p. 194)

"Je ne veux pas d'un gadget néocolonial où l'on repeint simplement la façade en noir. » (p. 195)

9. En juin 1988, au sortir de Matignon, lorsque Jean-Marie Tjibaou vint rencontrer les militants du soutien kanak à l'AISDPK (Association Information et soutien aux droits du peuple kanak) au Centre international de culture populaire (CICP) rue de Nanteuil, il nous avait fait part de la même crainte car il n'avait pas obtenu tout ce qu'il souhaitait. Mais une chose décisive pour lui était l'obtention de l'amnistie des militants kanak et donc la sortie de prison des Kanak de l'affaire d'Ouvéa. 
Dans la postface "Retour de Maré » (pp. 199 204), Walles Kotra revient, dans ses échanges avec Nidoïsh, sur :

" une interrogation qui [l]e taraude en plein ciel Et si Nidoïsh Naisseline était le dernier grand chef kanak ? [...] Nidoïh Naisseline n'annonce-t-il pas à sa manière la fin de la civilisation kanak ? La question mérite d'être posée de manière abrupte. [...] Au-delà de Naisseline et même plus largement de l'existence ou non des chefs en Calédonie, la question, la vraie question, est donc celle de l'avenir et de la survie de la culture kanak. » (pp. 200-201)

Cela n'est pas sans nous évoquer la "reformulation permanente " de Jean-Marie Tjibaou, comme nous le dit Nidoïsh lui-même (p. 197).

Cet ouvrage, que l'on doit ranger dans la liste à présent conséquente des livres biographiques portant sur des personnalités calédoniennes et $\operatorname{kanak}^{10} \mathrm{du} \mathrm{xx} \mathrm{x}^{\mathrm{e}}$ siècle, est important à plus d'un titre dans cette période pré-réfendaire sur l'avenir institutionnel de la Nouvelle-Calédonie. Il est un témoignage de toute une époque, qui peut permettre aux jeunes lecteurs kanak et calédoniens notamment, d'entrer de plein pied dans leur histoire. Car, en revenant à travers la vie de Nidoïsh Naisseline sur une grande partie de l'histoire politique coloniale de ce pays, on peut s'interroger à juste titre sur son avenir au moment où, quand j'écris ces lignes à l'automne 2017 et avant le comité de suivi des accords de début novembre, certains de la droite s'étaient alliés largement pour gagner les référendums en votant contre l'indépendance ${ }^{11}$. On aurait pu craindre alors que cela ne reparte pour un tour, les deux camps risquant de s'affirmer de plus en plus l'un contre l'autre et, après trente ans d'accords et beaucoup de mains tendues côté indépendantiste, finalement, on aurait pu se retrouver à la case départ ! Il semble que le comité des signataires ait réussi à dépasser cela. Reste maintenant à convaincre largement les électeurs en Nouvelle-Calédonie.

\section{RÉFÉRENCES CITÉES}

LeBLIC Isabelle, 1993. Les Kanak face au développement. La voie étroite, Grenoble, Presses universitaires de Grenoble, avec l'ADCK (Nouméa).

—, 2003. De la démocratie à la base : coutume et militantisme kanak dans les années 19851986, suivi de : Généalogie du paysage politique en Nouvelle-Calédonie (1946-1988). J.-M. Regnault (éd.), François Mitterrand et les territoires français du Pacifique (19811988). Mutations, drames et recompositions; enjeux internationaux et franco-français, Paris, Les Indes savantes, pp. 311-317 et 318-319 (<hal-00201023>).

Mokaddem Hamid, 2017. Yeiwéné Yeiwéné: Construction et Révolution de Kanaky (Nouvelle-Calédonie), La courte échelle / éditions transit, Kanaky-Calédonie 6.

NaIsSELINE Nidoïsh, 2011 (16 mars). Nidoïsh Naisseline veut conjuguer l'identité kanak au présent, Les Nouvelles calédoniennes (GIPTA500-7NAISSELINE.pdf).

Trépied Benoît, 2017. Les échos du passé. Enquête sur les débuts du Palika à Koné (NouvelleCalédonie, années 1970), Journal de la Société des Océanistes 144-145, pp. 239-252 (ce volume).

10. Un ouvrage sur Yeiwene Yeiwene vient de paraître (Mokaddem, 2017) et nous en rendrons compte dans nos colonnes prochainement.

11. Voir la plateforme "Gomès-Frogier-Yanno-Vittori » et la demande de Sonia Backes et des Républicains d'élargir cette alliance à une union sacrée de tous les loyalistes (http://www.dnc.nc/une-union-sacree-des-loyalistes-plus-large-quela-plateforme/). 